\title{
Chemotherapeutic cardiotoxicity is associated with elevated $\beta 1$-adrenergic receptor density
}

Manveen K Gupta, PhD, MBA*, Elizabeth E. Martelli, MBA, Kate T. Stenson, BS, and Sathyamangla V. Naga Prasad, PhD*

Department of Cardiovascular and Metabolic Sciences, Lerner Research Institute, Cleveland Clinic, Cleveland, OH, 44195

Short Title: Cardiotoxicity and $\beta 1 \mathrm{AR}$ upregulation

\section{*Address correspondence to:}

Sathyamangla V. Naga Prasad, PhD, FAHA or Manveen K. Gupta, PhD MBA

prasads2@ccf.org or guptam3@ccf.org

Department of Cardiovascular Medicine

Lerner Research Institute

Cleveland Clinic

9500 Euclid Avenue

Cleveland, OH, 44195

Tel: 2164443734

Fax: 2164458204

No industry relationship to disclose

Manuscript Category: Short Communication 


\section{Highlights}

In contrast to downregulation of $\beta 1$-adrenergic receptors ( $\beta 1 \mathrm{AR})$ in end-stage human heart failure, anthracycline cardiotoxicity-mediated failure is associated with selective increase in $\beta 1$ AR density.

ERBB2 inhibitor (AG825) treatment in mice results in cardiac dilation and selective rise in $\beta 1 \mathrm{AR}$ density showing that increased $\beta 1 \mathrm{AR}$ density in the heart could be a common mechanism underlying cardiotoxicity. 


\begin{abstract}
Objective: To understand the underlying pathways that promote cardiotoxicity following chemotherapy.
\end{abstract}

Background: Anthracyclines are associated with cardiotoxicity which could be potentiated with use of complementary agents (like anti-ERBB2 inhibitors) which together afford robust antineoplastic effects. Anthracyclines lead to oxidative stress and thought to induce cardiotoxicity. However, interventions reducing oxidative stress in patients have been unsuccessful suggesting mechanisms beyond oxidative stress. Despite $\beta$-adrenergic receptors ( $\beta$ ARs) being key regulators of cardiac function, nothing is known about their role in chemotherapy-mediated cardiotoxicity.

Methods: $\beta 1$ and/or $\beta 2$-AR density was assessed in end-stage human heart failure patient samples either due to anthracycline cardiotoxicity or non-anthracycline dilated cardiomyopathy (DCM). Since ERBB2 inhibition is integral to overall chemotherapeutic arsenal, we assessed $\beta 1$ - and/or $\beta 2$-AR density, cardiac function by echocardiography and immunohistochemistry in mice following ERBB2-specific inhibitor AG825.

Results: Selective increase in cardiac $\beta 1 \mathrm{AR}$ density is observed in end-stage human heart failure patient samples due to anthracycline cardiotoxicity as well as in ERBB2 inhibitor-treated mice.

Conclusions: Elevated $\beta 1 \mathrm{AR}$ density may be the key common underlying mechanism which is altered in response to chemotherapy promoting cardiac dilation of otherwise healthy hearts. 
Key words: Adriamycin cardiotoxicity, Epidermal growth factor receptor family, Cardiac dilation, ERBB2 inhibition, Beta-adrenergic receptor

Abbreviations: Epidermal growth factor receptor - EGFR; Erythroblastic oncogene B - ERBB2;

Dilated Cardiomyopathy - DCM, beta-adrenergic receptor - $\beta$ AR. 


\section{Introduction}

Anthracyclines have been the main stay therapy for cancer but, is associated with significant cardiotoxicity $^{1,2}$. It is estimated that $\sim 9 \%$ of the patients on anthracyclines will display cardiotoxicity ranging from dilated cardiomyopathy (DCM), congestive heart failure, atrial fibrillation to subclinical structural changes reflecting patient susceptibility ${ }^{1}$. Retrospective studies have shown that majority of the patients develop cardiac dysfunction/failure spanning the New York Heart Association (NYHA) Classes I through IV heart failure classification ${ }^{3}$. Given that conventional anthracyclines causes cardiotoxicity, next generation of targeted cancer therapeutics were generated that had specific pharmacologic action like trastuzumab (humanized antibody directed against HER2/ERBB2 (Epidermal growth factor receptor 2/Erythroblastic oncogene B, ERBB2), sunitinib (Vascular endothelial growth factor (VEGF) inhibitor), dasatinib (Break point cluster (Bcr) chimera with Abl tyrosine kinase (BCR-ABL) inhibitor) to name a few $^{1}$. However, majority of these targeted therapies are still associated with cardiotoxicity ${ }^{1}$. Despite the knowledge that chemotherapy increases oxidative stress resulting in lipid peroxidation and mitochondrial dysfunction, use of anti-oxidants in patients was not cardioprotective $^{4}$. This shows that additional mechanisms underlie chemotherapy-induced cardiotoxicity.

$\beta$-adrenergic receptors ( $\beta$ ARs) are powerful regulators of cardiac function, but less is known about their roles in cardiac remodeling following cardiotoxicity. $\beta$ AR family is subdivided into $\beta 1 \mathrm{AR}, \beta 2 \mathrm{AR}$ and $\beta 3 \mathrm{AR}^{5}$ based on their pharmacological properties with $\beta 1 \mathrm{AR}$ being the major regulator of cardiac function ${ }^{5}$. Consistently, end-stage human heart failure patient samples are characterized by selective downregulation of $\beta 1$ ARs associated with DCM and heart failure ${ }^{6}$. Given the key role of $\beta 1 \mathrm{ARs}$ in cardiac function, we assessed whether alterations in $\beta 1 \mathrm{AR}$ density underlies cardiotoxicity with anthracycline (using end-stage anthracycline-mediated human heart failure patient samples) or ERBB2 targeting (by treating mice with ERBB2-specific inhibitor (AG825)) representing a common mechanism for deleterious cardiac remodeling.

\section{Methods:}

Human heart failure samples: Procurement of the end-stage human heart failure samples were approved by the institutional IRB. All the patient samples (non-failing, DCM or DCM due 
Adriamycin cardiotoxicity) were de-identified. Gender, age and clinical characteristics are shown in Table 1.

Plasma membrane isolation and $\beta A R$ density: Plasma membranes were isolated as described previously ${ }^{7}$. Pellet representing the plasma membrane fraction was resuspended in 75 mM Tris$\mathrm{HCl} \mathrm{pH}$ 7.5, $2 \mathrm{mM}$ EDTA, and $12.5 \mathrm{mM} \mathrm{MgCl}_{2}$ for assess $\beta A R$ density. $\beta A R$ density was determined by incubating $20 \mu \mathrm{g}$ of the membranes from mouse or end-stage human heart failure samples with saturating concentrations of ${ }^{125}$ I-Cyanopindolol. $100 \mu \mathrm{M}$ propranolol was used for assessing non-specific binding and $\beta 2 \mathrm{AR}$ specific antagonist ICI 181,551 was used for determining $\beta 2 \mathrm{AR}$ density. These were subtracted from the non-specific propranolol values to determine $\beta 1 \mathrm{AR}$ density.

Experimental Animals: Male and female C57BL/6 mice from Jackson labs were used for the studies. Animals were handled according to the approved protocols and animal welfare regulation of IACUC at Cleveland Clinic following the approved NIH guidelines.

ERBB2 inhibitor studies: ERBB2-specific inhibitor AG 825 dissolved in DMSO and diluted in saline was used at a final concentration of $1 \mathrm{mg} / \mathrm{kg} /$ day through min-osmotic pump (ALZET model 2002) for 14 days.

Echocardiography: Echocardiography was performed on anesthetized mice using a VEVO 770/VEVO 2100 (VISUALSONICS) echocardiographic machine as previously described ${ }^{8}$. Mmode recording was used to obtain functional parameters.

Statistics: Data are expressed as mean \pm SEM. Statistical comparisons were performed using an unpaired Student's t-test for two samples comparison (like binding studies) and analysis of variance (ANOVA) was carried out for multiple comparisons (like paired echocardiography analysis). Post-hoc analysis was performed with a Scheffe’s test. For analysis, a value of * $\mathrm{p}<$ 0.05 was considered significant. 


\section{RESULTS AND DISCUSSION}

Adriamycin-mediated human heart failure is associated with increased $\beta 1 \mathrm{AR}$ - It is not known whether anthracyclines which mediates deleterious cardiac remodeling affects cardiac $\beta$ ARs. Multiple studies including ours have shown that end-stage human heart failure dilated cardiomyopathy (DCM) patient samples are characterized by significant down-regulation of $\beta 1 \mathrm{ARs}^{5,6,9}$. Radio-ligand binding studies were performed to test whether $\beta \mathrm{ARs}$ are altered in the end-stage human heart failure samples due to anthracycline (Adriamycin) cardiotoxicity or DCM patient samples [patient characteristics: Table 1]. Consistently, downregulation of $\beta 1 \mathrm{ARs}$ was observed in end-stage DCM samples ${ }^{6,9}$ [Fig. 1A, grey bar, left panel] but surprisingly, there was significant increase in $\beta 1 \mathrm{AR}$ density in the human heart failure samples due to Adriamycin cardiotoxicity [Fig.1A, black bar, left panel]. Furthermore, $\beta 1 \mathrm{AR}$ density in the Adriamycin cardiotoxic human heart failure samples was significantly increased even compared to the nonfailing controls [Fig.1A white vs. black bar, left panel]. However, $\beta 2 \mathrm{AR}$ density in the Adriamycin cardiotoxic heart failures samples was not appreciably different than non-failing controls [Fig. 1A, middle panel, white vs. black bar] which was significantly downregulated in end-stage DCM samples [Fig.1A, middle panel, grey bar]. While DCM heart failure samples showed significant downregulation of $\beta A R s$ [Fig. 1A, right panel, grey bar], the Adriamycin cardiotoxic heart failure samples showed minimal loss in $\beta A R$ density compared to non-failing samples [Fig.1A, right panel, black vs. white bars]. These observations show that the chemotherapeutic agents may induce cardiac dilation/heart failure in otherwise healthy hearts by upregulating $\beta 1 \mathrm{ARs}$, in contrast to heart failure etiology characterized by downregulation of $\beta 1 \mathrm{ARs}^{5,6,9}$. Consistently, studies have shown that $\beta 1 \mathrm{AR}$ signaling is pro-apoptotic ${ }^{10}$ and cardiomyocyte-specific overexpression of $\beta 1 \mathrm{AR}$ results in cardiac dilation and accelerated heart failure ${ }^{5}$.

ERBB2 inhibition results in increase of $\beta 1 \mathrm{AR}$ density - Since targeted chemotherapies like sunitinib, dasatinib or trastuzumab also leads to DCM, we tested whether alterations in the cardiac $\beta 1$ ARs could be a common underlying mechanism for cardiotoxicity. ERBB2 inhibition was performed by using AG 825 (a selective ERBB2 inhibitor) instead of trastuzumab/Herceptin as they are humanized antibodies which may initiate confounding immune response in mice. Radio-ligand binding studies showed modest yet, significant increase in $\beta 1 \mathrm{AR}$ density after 
ERBB2 inhibition in mice [Fig. 1B] with no appreciable changes in $\beta 2 A R$ density [Fig 1B]. Consistent with the increased $\beta 1 \mathrm{AR}$ density, there was significant elevation in total $\beta \mathrm{AR}$ density [Fig.1B] following AG 825 administration. Interestingly, there was mild yet significant cardiac dysfunction following AG 825 treatment as observed by M-mode echocardiography and key measures of cardiac function \% FS (fraction shortening) and \% EF (ejection faction) [Fig. 1 C, D \& E]. Furthermore, H \& E staining reflected cardiac dilation following AG 825 treatment [Fig. 1F]. Similar to our previous studies ${ }^{11}$, appreciable cardiac dysfunction was not observed in females while males are susceptible to AG 825 within two weeks, an observation that is consistent with the role of estrogens in cardio-protection ${ }^{12}$. However, both males and females showed increase in $\beta 1 \mathrm{AR}$ density indicating that molecular events underlying the future deleterious outcomes precede the physiologic measures of cardiac function. Thus, increasing the timeline of AG 825 administration may lead to cardiac dysfunction in females. These findings indicate that chemotherapeutics (targeted/untargeted) may alter the homeostatic regulation of $\beta 1 \mathrm{AR}$ resulting in selective increase of $\beta 1 \mathrm{AR}$ density that promotes deleterious cardiac remodeling ${ }^{5,10}$ [Fig. 1G]. These observations set the foundation to the conceptual idea that use of selective $\beta 1 \mathrm{AR}$ blockers may ameliorate cardiac dysfunction insulating the heart from chemotherapeutic cardiotoxicity.

Acknowledgements: The authors would like to thank Wendy Sweet and Dr. Christine S. Moravec for providing us end-stage DCM and anthracycline cardiotoxicity human heart failure samples. This work is in part, supported by Postdoctoral Fellowship Grant, AHA, 10POST3610049 (MKG).

Disclosure: The authors of the paper have no conflicts and nothing to disclose. 
1. Herrmann J. Adverse cardiac effects of cancer therapies: cardiotoxicity and arrhythmia. Nat Rev Cardiol. 2020;17:474-502.

2. Saleh Y, Abdelkarim O, Herzallah K and Abela GS. Anthracycline-induced cardiotoxicity: mechanisms of action, incidence, risk factors, prevention, and treatment. Heart Fail Rev. 2020.

3. Swain SM, Whaley FS and Ewer MS. Congestive heart failure in patients treated with doxorubicin: a retrospective analysis of three trials. Cancer. 2003;97:2869-79.

4. Octavia Y, Tocchetti CG, Gabrielson KL, Janssens S, Crijns HJ and Moens AL.

Doxorubicin-induced cardiomyopathy: from molecular mechanisms to therapeutic strategies. $J$ Mol Cell Cardiol. 2012;52:1213-25.

5. $\quad$ Engelhardt S. Beta-adrenergic receptors in heart failure. Heart Fail Clin. 2005;1:183-91.

6. Perrino C, Schroder JN, Lima B, Villamizar N, Nienaber JJ, Milano CA and Naga Prasad SV. Dynamic regulation of phosphoinositide 3-kinase-gamma activity and beta-adrenergic receptor trafficking in end-stage human heart failure. Circulation. 2007;116:2571-9.

7. Vasudevan NT, Mohan ML, Gupta MK, Hussain AK and Naga Prasad SV. Inhibition of protein phosphatase 2A activity by PI3Kgamma regulates beta-adrenergic receptor function. $\mathrm{Mol}$ Cell. 2011;41:636-48.

8. Vasudevan NT, Mohan ML, Gupta MK, Martelli EE, Hussain AK, Qin Y, Chandrasekharan UM, Young D, Feldman AM, Sen S, Dorn GW, 2nd, Dicorleto PE and Naga Prasad SV. Gbetagamma-independent recruitment of G-protein coupled receptor kinase 2 drives tumor necrosis factor alpha-induced cardiac beta-adrenergic receptor dysfunction. Circulation. 2013;128:377-87.

9. Insel PA and Hammond HK. Beta-adrenergic receptors in heart failure. J Clin Invest. 1993;92:2564.

10. Zhu WZ, Wang SQ, Chakir K, Yang D, Zhang T, Brown JH, Devic E, Kobilka BK, Cheng $\mathrm{H}$ and Xiao RP. Linkage of beta1-adrenergic stimulation to apoptotic heart cell death through protein kinase A-independent activation of Ca2+/calmodulin kinase II. J Clin Invest. 2003;111:617-25.

11. Sysa-Shah P, Tocchetti CG, Gupta M, Rainer PP, Shen X, Kang BH, Belmonte F, Li J, Xu Y, Guo X, Bedja D, Gao WD, Paolocci N, Rath R, Sawyer DB, Naga Prasad SV and Gabrielson K. Bidirectional cross-regulation between ErbB2 and beta-adrenergic signalling pathways. Cardiovasc Res. 2016;109:358-73.

12. Cadeddu Dessalvi C, Pepe A, Penna C, Gimelli A, Madonna R, Mele D, Monte I, Novo G, Nugara C, Zito C, Moslehi JJ, de Boer RA, Lyon AR, Tocchetti CG and Mercuro G. Sex differences in anthracycline-induced cardiotoxicity: the benefits of estrogens. Heart Fail Rev. 2019;24:915-925. 
Figure: A, Radio-ligand binding in non-failing (NF) human heart (open bar) ( $\mathrm{n}=5)$, dilated cardiomyopathy (DCM) (grey) $(n=5)$ or Adriamycin-mediated heart failure (AHF) (black) $(n=5)$. Left panel: $\beta 1$ AR density. $\# p<0.001$ vs. NF and AHF; ${ }^{*} p<0.001$ vs. NF and DCM; Middle: $\beta 2 A R$ density. $\# p<0.005$ vs. NF and AHF; Right: Total $\beta A R$ density. $\# p<0.005$ vs. NF and AHF. B, $\beta 1$ AR density (left panel), $\beta 2$ AR density (middle) and total $\beta A R$ density measured by radioligand binding on plasma membranes following Vehicle (VEH) or ERBB2 inhibitor $(\mathrm{n}=8)$, ${ }^{*} \mathrm{p}<0.0001$ vs. Vehicle. Echocardiography for the VEH or ERBB2 inhibitor treated mice $(\mathrm{n}=11)$ (C) and associated functional assessment by \% fractional shortening (\%FS) (D) and \% ejection fraction (EF) (E), ${ }^{*} \mathrm{p}<0.005$ vs. other sample measures. F, Transverse heart section stained with $\mathrm{H} \& \mathrm{E}$ following VEH or ERBB2 inhibitor. Scale bar $1000 \mu \mathrm{m}$. G, Proposed schematic showing that anthracycline/ERBB2 inhibitor selectively elevates $\beta 1 \mathrm{AR}$ density and its signaling may underlie cardiotoxicity. 
Table 1: Patient Characteristics

\begin{tabular}{|c|c|c|c|c|c|c|c|c|}
\hline & Age & Gender & Race & $\begin{array}{l}\text { Cause } \\
\text { of death }\end{array}$ & Diagnosis & \% LVEF & $\begin{array}{c}\text { Medications } \\
\text { prior to the event }\end{array}$ & $\begin{array}{l}\text { Acutely administered } \\
\text { medications }\end{array}$ \\
\hline \multicolumn{9}{|c|}{ NON-FAILING } \\
\hline 1 & 57 & $\mathrm{~F}$ & W & CVA & - & ND & - & DOP \\
\hline 2 & 61 & $\mathrm{~F}$ & W & CVA & - & 50 & - & DOP, NE \\
\hline 3 & 60 & $\mathrm{~F}$ & W & Sub-AB & - & 65 & - & DOB, NE, PHE, THY \\
\hline 4 & 62 & $\mathrm{~F}$ & W & CVA & - & ND & - & NE, PHE, VP \\
\hline 5 & 64 & $\mathrm{~F}$ & W & CVA & - & 70 & - & NE, THY, VP \\
\hline 6 & 62 & $\mathrm{~F}$ & $\mathrm{~B}$ & CVA & - & 67 & - & DOP, NE, THY, VP \\
\hline \multicolumn{9}{|c|}{ DILATED CARDIOMYOPATHY (DCM) HEART FAILURE } \\
\hline 1 & 60 & $\mathrm{~F}$ & W & - & DCM-Viral & 10 & AM, DIG, DOB & - \\
\hline 2 & 61 & $\mathrm{~F}$ & W & - & DCM & 10 & CARV, DIG, RAMP & - \\
\hline 3 & 63 & $\mathrm{~F}$ & $\mathrm{~B}$ & - & DCM & 18 & CARV, DIG & - \\
\hline 4 & 62 & $\mathrm{~F}$ & $\mathrm{~W}$ & - & DCM & 14 & CARV, & - \\
\hline 5 & 63 & $\mathrm{~F}$ & W & - & DCM & 31 & CARV, QUIN & - \\
\hline 6 & 58 & $\mathrm{~F}$ & $\mathrm{~B}$ & - & DCM & 18 & AM, DIG, MIL & - \\
\hline \multicolumn{9}{|c|}{ CHEMOTHERAPEUTIC (ADRIAMYCIN) HEART FAILURE } \\
\hline 1 & 62 & $\mathrm{~F}$ & W & - & DCM-Chemo & 12 & CAPT, DIG, MIL & - \\
\hline 2 & 63 & $\mathrm{~F}$ & B & - & DCM-Chemo & 10 & CARV, LOS & - \\
\hline 3 & 63 & $\mathrm{~F}$ & $\mathrm{~W}$ & - & DCM-Chemo & 8 & DIG, LIS, MIL & - \\
\hline 4 & 58 & $\mathrm{~F}$ & W & - & DCM-Chemo & 19 & - & - \\
\hline 5 & 61 & $\mathrm{~F}$ & W & - & DCM-Chemo & ND & $\mathrm{AM}$ & - \\
\hline
\end{tabular}

ABBREVIATIONS: AM- Amiodarone, B- Black, CAPT- Captopril, CARV-Carvedilol, CVA- Cerebral vascular accident, DCMDilated cardiomyopathy, DOB- Dobutamine, DOP- Dopamine, F- Female, LIS- Lisinopril, LOS- Losartan, LVEF- Left ventricular ejection fraction, MIL- Milrinone, ND- Not determined, NE- Norepinephrine, PHE- Phenylephrine, QUIN- Quinapril, RAMPRamapril, Sub-AB- Sub-Arachnoid Bleed, THY- Levothyroxine, VP- Vasopressin, W- White. 
A Human heart samples

$\square$ Non-failing $\square D C M$ heart failure heart failure
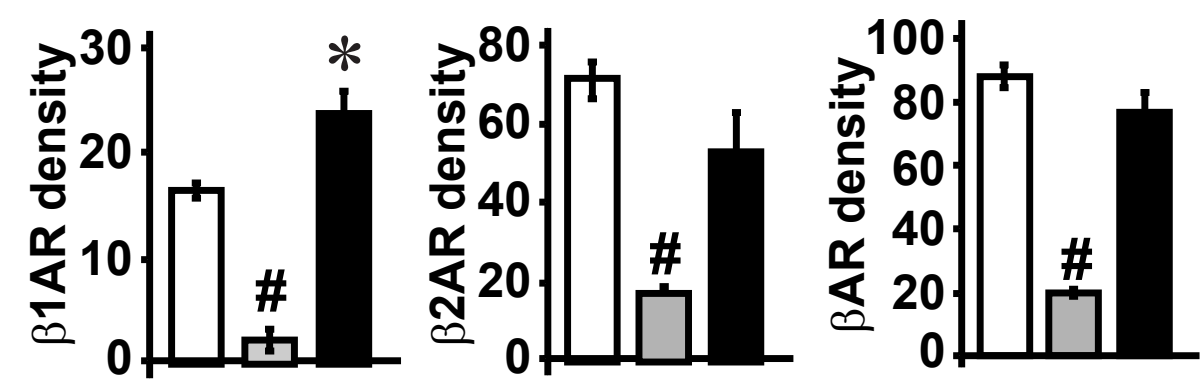

Adriamycin

B

ERRB2 inhibitor upregulates $\beta 1 A R$

口 VEH ERBB2 inhibitor
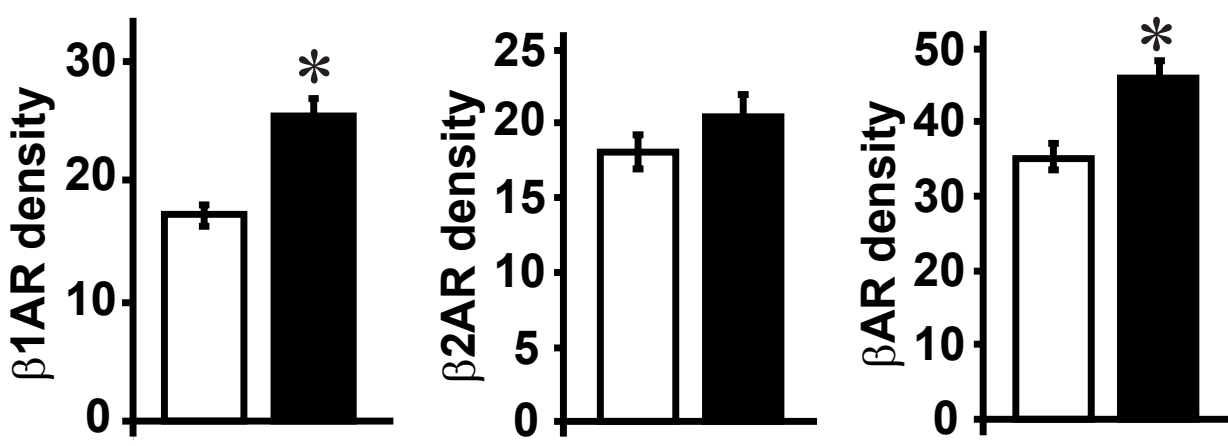

C

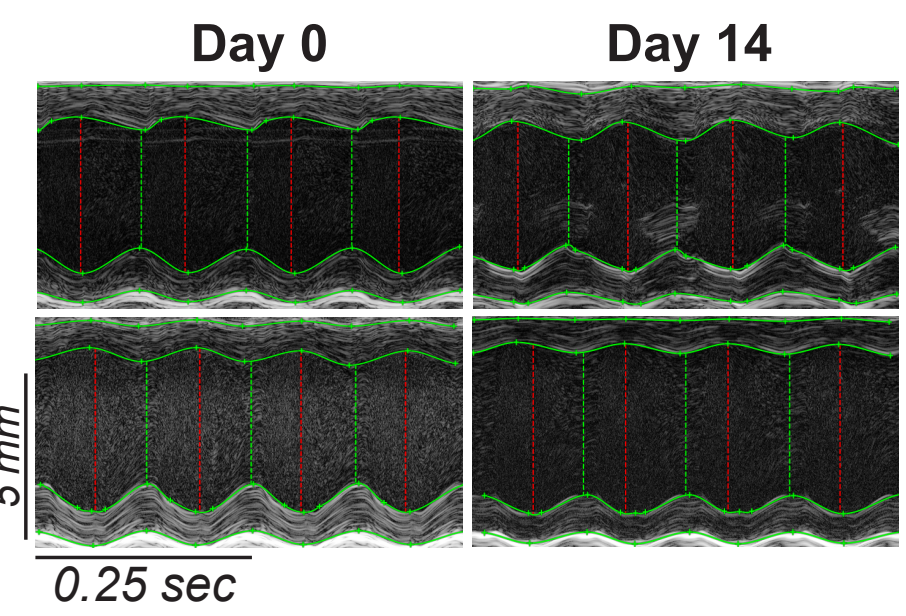

$\mathbf{F}$

ERBB2 inhibitor treatment (H \& E staining)
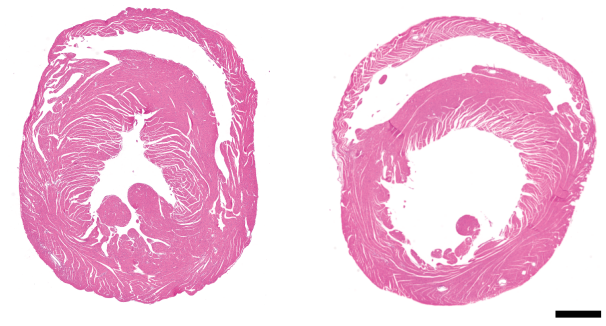

VEH ERRB2 Inhibitor
D $\mathrm{VEEH}$ Q ERRB2 inhibitor

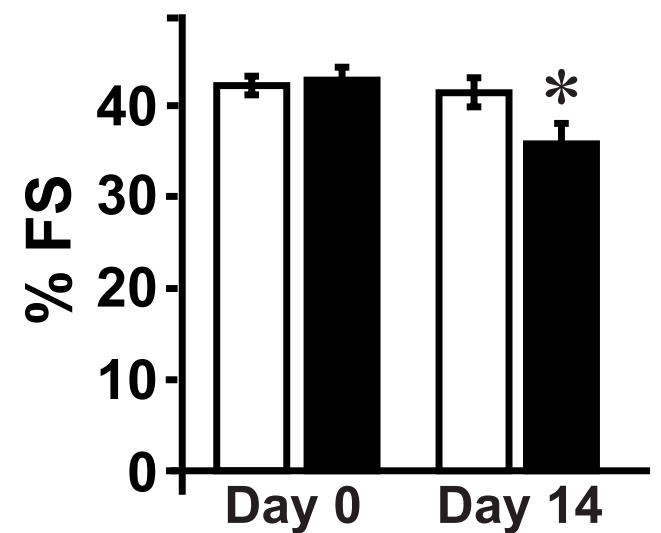

G

ERBB2 inhibitor

VEH

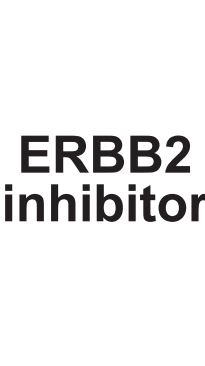

G Normal Physiology

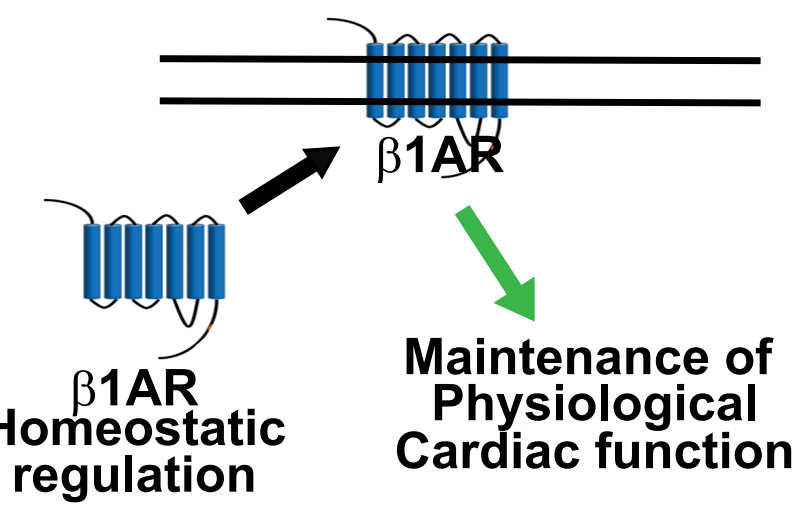

E

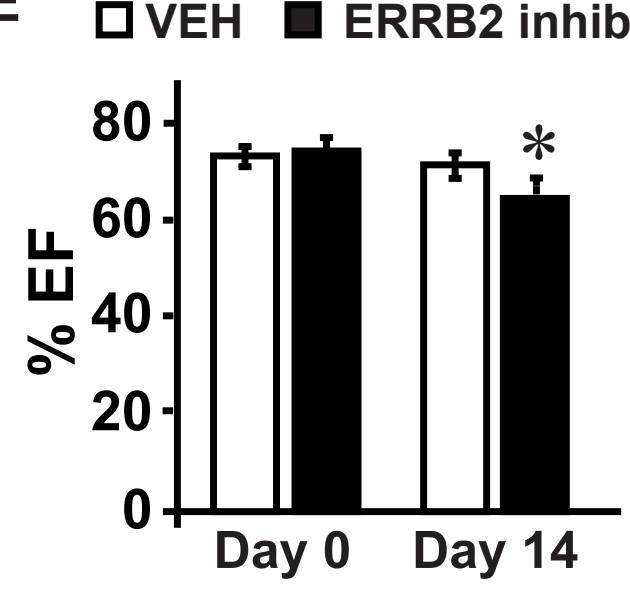

Increased $\beta 1 A R$ density with Chemotherapeutics

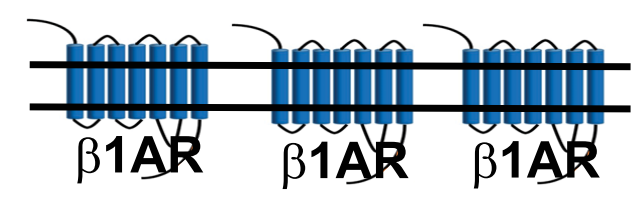

Anthracylines/ ERBB2 Inhibitors

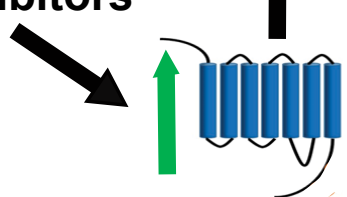

Cardiac Dilation 